\title{
The applications of pollen analysis in environmental monitoring in Akoko North- East Local Government Area of Ondo State, Nigeria
}

\author{
Ige Olugbenga Ebenezer * and Essien Benjamin Christopher \\ Department of Plant Science and Biotechnology, Adekunle Ajasin University, Akungba-Akoko, Ondo State, Nigeria.
}

Publication history: Received on 22 June 2019; revised on 10 July 2019; accepted on 15 July 2019

Article DOI: https://doi.org/10.30574/gscbps.2019.8.1.0113

\begin{abstract}
The use of pollen and spores in environmental studies is primarily in its application to the study of vegetational history and the relevance of atmospheric pollen content to the vegetation of a region is related to the palynomorphs produced in situ and those supplied from the surrounding ecological zones. Aeropalynological studies towards monitoring environmental change in Akoko North-East Local Government Area of Ondo State, Nigeria through pollen analysis was carried out in five randomly selected locations comprising of ten sampling sites to determine the pollen types present in the atmosphere of the study environment and the relationship between the airborne pollen assemblages with its vegetation. The palynomorphs were collected with Modified Tauber Sampler using simple random sampling technique and analyzed palynologically. All data were subjected to statistical analyses and results showed that 15,286 palynomorphs were documented of which 149 pollen types belonging to 67 angiospermic plant families were encountered. Three (3) were identified to family level, 48 to species level, 97 to generic level and one (1) unidentified. The presence of Empetrum nigrum, Linum sp. and Vitellaria paradoxa pollen indicates evidence of long distance transport. Pollen types from exotic plants and charred plant particles were part of the atmospheric palynomorphs content recorded as evidence of human impact. Pollen assemblages reflected tropical rainforest vegetation type despite high level of anthropogenic activities. Environmental monitoring through pollen rain analysis and adequate conservation measures through afforestation and prevention of annual bush burning are recommended for safety health and environmental sustainability in the studied area.
\end{abstract}

Keywords: Airborne palynomorphs; Environment; Pollen analysis; Vegetation

\section{Introduction}

The study of vegetation and the way in which it has been altered and developed in the course of time indicates past changes that have occurred in our terrestrial environment. Variations in climate and in the intensity of human activity in historic and prehistoric times have made their mark upon vegetation, and the plants themselves have left a record of these changes in the form of vast quantities of pollen grains which have survived in contemporary sediments [1].

The use of pollen and spores in environmental studies is primarily in its application to the study of vegetational history. Conclusion about climate and human disturbances could be deduced from such analysis and they are termed secondary deductions [2]. Fact gathered from such analysis could be useful to climatologists and oil explorationists among others [3]. Basically, pollen analysis is a technique for reconstruction of former vegetation by means of the pollen grains recovered from sediments. Since the pollen grain exine is resistant, it may have a long geological life once it is incorporated into sediment, but only if the grains avoid mechanical attrition and chemical changes such as oxidation [4].

\footnotetext{
${ }^{*}$ Corresponding author

E-mail address: olugbengaige@yahoo.com
} 
The relevance of atmospheric pollen content to the vegetation of a region is related to the palynomorphs produced in situ and those supplied from the surrounding ecological zones. Pollen and spores are generally produced in excess of the biological needs of the plants. Established observations have shown that anemophilous plants release large quantities of pollen into the air, while entomophilous taxa release smaller amounts. The behavioural patterns leads to differences in the quantity of pollen and spores of various taxa available in the atmosphere, and which of them can be trapped during sampling.

The widely dispersed pollen provides a broad picture of the surrounding vegetation of the areas in which they are produced. Even though sometimes, some of them are blown into these areas from distant places, experienced palynologists are most of the time able to detect these re-deposited pollen and spores by differences in preservation (e.g. colour, corrosion, wear), ecological or stratigraphical inconsistencies and other associated evidences of being redeposited [5].

The quantity and quality of palynomorphs, especially pollen grains and spores in the air at any given time depend largely on the plant and fungi producing them, the abundance of the plant communities, the nature of palynomorphs, the flowering or season of reproduction and the meteorological factors such as rainfall, humidity, temperature, wind speed and wind direction [6].

In recent years, many palynological studies have been carried out in West Africa. These studies have helped in reconstructing past vegetation types of different ecosystems as well as predicting the type of climatic conditions that prevailed during such periods [7-13].

The aim of the present study is to determine the pollen types present in the atmosphere of the study environment and the relationship between the airborne pollen assemblages with its vegetation.

\section{Material and methods}

\subsection{Description of the Study Area}

Akoko North-East is a Local Government Area in Ondo State, Nigeria. Its headquarters is in the town of Ikare. It has an area of $372 \mathrm{Km}^{2}$ and a population of 175,409 at the 2006 Census.

\subsection{Vegetation of the study area}

The natural vegetation of Ondo State is the high forest, composed of many varieties of hardwood timber such as Milicia excelsa, Antiaris africana, Terminalia superba, Lophira procera and Symphonia globulifera. In the northern districts, the vegetation consists of woody savanna featuring such tree species as Blighia sapida and Parkia biglobosa. The swamp flats are the domain of the fresh water swamp forests in the interior and the units of mangrove vegetation near the coast. The sand ridges are characterized by savanna and stunted rain forests taxa. Over most part of the state, the natural vegetation has been very much degraded as a result of human activities, the chief of which is based on the rotation of bush fallow system and lumbering. As a result, the original forest is now restricted to forest reserves. An important aspect of the vegetation of the Ondo State is the prevalence of tree crops. The major tree crops include Theobroma cacao (Cocoa), Cola nitida (Kola), Coffee arabica (Coffee), Hevea brazillensis (Rubber), Elaeis guineensis (Oil palms) and Citrus spp., cocoa being the most prevalent.

\subsection{Climate}

The climate of Ondo State is of the Tropical Rain Forest type, with distinct wet and dry seasons. In the south, the mean monthly temperature is $27^{\circ} \mathrm{C}$, with a mean monthly range of $2{ }^{\circ} \mathrm{C}$, while mean relative humidity is over seventy five percent. However, in the northern part of the state, the mean monthly temperature and its range are about $30^{\circ} \mathrm{C}$ and $6^{\circ} \mathrm{C}$ respectively. The mean monthly relative humidity is less than seventy percent. In the south, rain falls throughout the year, but the three months of November, December and January may be relatively dry. The mean annual total rainfall exceeds 2000 millimetres. However, in the north, there is a marked dry season from November to March when little or no rain falls. The total annual rainfall in the north, therefore, drops considerably to about 1800 millimetres.

\subsection{Sampling techniques}

A total of 10 locations were selected within 5 representative communities in Akoko North-East Local Government Area of Ondo State, Nigeria as sampling sites. The sampling sites for the study was purposely [14] selected to reflect 
(represent) as far as possible the Local Government Area of the study. In choosing the sites, consideration was also given to urbanization, accessibility, and safety of the sampling (experimental materials) instruments among others.

At each site, a pollen trap (Modified Tauber Sampler) was mounted according to the methods of Tauber [15, 16], Pardoe et al. [17] and Giesecke et al. [18]. Prior to this, a mixture of glycerol (65 ml), formalin $(30 \mathrm{ml})$ and phenol (5 $\mathrm{ml}$ ) was poured into each of the trap. The positions of the traps at various locations were recorded using a Global Position System (GPS). The solutions in the trap prevented the palynomorphs from drying up, kill insects and also prevented the decay of dead organisms. The trap was left to stand throughout the duration of the study period. Fortnightly of each month, solution collection was done. The traps were washed with water to remove any contaminants and were then recharged with the above mentioned chemical solution. This procedure was repeated bimonthly from October 2016 to December 2017 (dry season and rainy seasons' samples) for one year. The palynomorphs were recovered through centrifugation at 2000 r.p.m (revolution per minute) for 5 minutes and supernatant decanted each time. The precipitates were washed twice with distilled water and recovered through centrifugation. The sediments were treated with glacial acetic acid to remove water before acetolysis [2, 19]. The recovered precipitates were washed with glacial acetic acid, and finally washed twice with distilled water, centrifuged each time and decanted. The recovered palynomorphs were stored in a plastic vials in glycerin and ethanol solution $(2: 1)$.

The palynomorphs were analysed palynologically and microscopically with Olympus microscope at $\mathrm{x} 400$ magnification for counting and Leica microscope at x1000 magnification for detailed morphological studies. Palynomorphs identification, counting and classification was done with the help of reference descriptions and photomicrographs from Agwu and Akanbi [19], Bonnefille and Riollet [20], Kremp and Kawasaki [21], Sowunmi [22] and Shubharani et al. [23]. In addition, prepared slides of pollen samples in the Palynological Research Unit; Department of Plant Science and Biotechnology, Adekunle Ajasin University, Akungba- Akoko, Nigeria were used.

\section{Results and Discussions}

The results of the study of airborne palynomorphs precipitation showed that the following particulate entities: pollen grains, spores of pteridophytes and fungi, diatom frustules, fungal hyphae, arthropods (insects and insect parts), plant tissues and bryophyte parts as well as charred plant particles were recorded at varying quantities and qualities (Table 1). Comparative analysis showed that pollen grains constituted the highest counts, followed by charred plant particles, arthropods, diatom frustules, plant tissues, bryophyte particles and fungal hyphae (Table 1). The distribution of palynomorphs abundance is presented in Fig. 1. Absolute palynomorphs counts across the study locations are given in Table 2 while the Mean pollen grains recorded at the study locations are shown in Table 3. Statistical analysis shows that there was significant difference in the mean pollen grains counts recorded across the study locations. Photomicrograph of some selected palynomorphs is presented in Fig. 2.

Table 1 Summary of the palynomorphs recorded in the study (Oct., 2016 - Sept., 2017)

\begin{tabular}{lllllll}
\hline Pollen types/ Locations & Akunnu & Auga & Iboropa & Ikare & Ise & Total \\
\hline Pollen grains & 2,664 & 2,338 & 2,587 & 2,130 & 1,923 & 11,642 \\
Pteridophyte spores & 47 & 62 & 58 & 44 & 25 & 236 \\
Algal cysts & 5 & 3 & 7 & 3 & 9 & 27 \\
Athropods/ parts & 140 & 468 & 51 & 168 & 274 & 1,101 \\
Bryophyte & 5 & 0 & 0 & 0 & 1 & 6 \\
Charred plant tissue & 457 & 124 & 396 & 133 & 184 & 1,294 \\
Diatom frustules & 149 & 276 & 125 & 186 & 199 & 935 \\
Dinoflagellate cysts & 9 & 13 & 7 & 5 & 0 & 34 \\
Fungal hyphae & 0 & 0 & 3 & 0 & 0 & 3 \\
Plant tissues & 0 & 0 & 1 & 0 & 7 & 8 \\
TOTAL & $\mathbf{3 , 4 7 6}$ & $\mathbf{3 , 2 8 4}$ & $\mathbf{3 , 2 3 5}$ & $\mathbf{2 , 6 6 9}$ & $\mathbf{2 , 6 2 2}$ & $\mathbf{1 5 , 2 8 6}$ \\
\hline
\end{tabular}


Table 2 Absolute palynomorph counts across the study locations

\begin{tabular}{|c|c|c|c|c|c|c|c|}
\hline Pollen types/ Locations & Plant Family & Akunnu & Auga & Iboropa & Ikare & Ise & Total \\
\hline Abelmoschus esculenta & Malvaceae & - & - & - & 5 & - & 5 \\
\hline Acacia senegal & Mimosoideae & - & 4 & 3 & - & - & 7 \\
\hline Acacia sp. & Mimosoideae & - & - & - & - & 1 & 1 \\
\hline Acalypha sp. & Euphorbiaceae & - & - & 1 & - & - & 1 \\
\hline Adenanthera pavonina & Mimosoideae & 2 & - & 2 & 1 & - & 5 \\
\hline Afzelia africana & Caesalpinoideae & - & 2 & 5 & - & - & 7 \\
\hline Albizia sp. & Mimosoideae & - & 5 & 14 & 2 & 6 & 27 \\
\hline Albzzia zygia & Mimosoideae & 5 & 21 & - & 13 & 17 & 56 \\
\hline Alchornea cordifolia & Euphorbiaceae & 154 & 36 & 56 & 39 & 107 & 392 \\
\hline Allopyhllus sp. & Sapindaceae & 2 & - & - & - & - & 2 \\
\hline Alstonia boonei & Apocynaceae & 2 & 2 & 1 & - & 2 & 7 \\
\hline Amaranth. / Cheno. & & 45 & 7 & 33 & 26 & 19 & 130 \\
\hline Anacardium occidentale & Anacardiaceae & - & - & 6 & - & 2 & 8 \\
\hline Aneilema sp. & Commelinaceae & 3 & & - & - & - & 3 \\
\hline Anisotes sp. & Acanthaceae & - & - & 2 & - & - & 2 \\
\hline Annona senegalensis & Annonaceae & 3 & - & - & - & 8 & 11 \\
\hline Anthocleista djalonensis & Loganiaceae & 2 & - & 2 & - & - & 4 \\
\hline Anthrocaryon micraster & Anacardiaceae & - & - & 1 & - & - & 1 \\
\hline Antidesma sp. & Phyllanthaceae & 8 & 12 & 21 & 7 & 14 & 62 \\
\hline Aspilia africana & Asteraceae & 10 & 14 & 17 & 20 & 39 & 100 \\
\hline Aubrevillea kerstingii & Mimosoideae & - & 5 & - & - & - & 5 \\
\hline Azadirachta indica & Meliaceae & 5 & 6 & 1 & 2 & 5 & 19 \\
\hline Baphia sp. & Papilionoideae & - & 2 & 2 & - & 2 & 6 \\
\hline Barteria nigritiana & Passifloraceae & - & 1 & - & 2 & - & 3 \\
\hline Berlinia grandifolia & Caesalpinoideae & 4 & 5 & - & - & 3 & 12 \\
\hline Blighia unijugata & Sapindaceae & - & 5 & - & 3 & - & 8 \\
\hline Bombax buonopozense & Bombacaceae & 7 & 10 & 7 & 3 & 6 & 33 \\
\hline Borreria sp. & Rubiaceae & 2 & - & - & - & - & 2 \\
\hline Brachystegia eurycoma & Caesalpinoideae & 2 & 2 & 1 & - & - & 5 \\
\hline Bridelia ferruginea & Phyllanthaceae & 2 & 2 & 2 & - & - & 6 \\
\hline Calycobolus sp. & Convolvulaceae & 1 & - & - & - & - & 1 \\
\hline Canarium schweinforthii & Burseraceae & - & 2 & - & 3 & - & 5 \\
\hline Canthium sp. & Rubiaceae & 5 & - & - & - & 3 & 8 \\
\hline Capparis sp. & Capparaceae & - & 3 & 1 & - & - & 4 \\
\hline Carica papaya & Caricaceae & 3 & - & - & 4 & - & 7 \\
\hline Casuarina equisetifolia & Casuarinaceae & 61 & 31 & 110 & 34 & 33 & 269 \\
\hline
\end{tabular}




\begin{tabular}{|c|c|c|c|c|c|c|c|}
\hline Ceiba pentandra & Bombacaceae & 2 & 2 & 1 & - & - & 5 \\
\hline Celtis sp. & Cannabaceae & 2 & 3 & 1 & - & - & 6 \\
\hline Citrus sp. & Rutaceae & 4 & 7 & 3 & 2 & - & 16 \\
\hline Cleome sp. & Capparaceae & 2 & - & - & - & - & 2 \\
\hline Cnestis ferruginea & Connaraceae & 2 & 1 & 3 & - & - & 6 \\
\hline Cochlospermum planchonii & Cochlospermaceae & 5 & 2 & - & 5 & 1 & 13 \\
\hline Cocos nucifera & Arecaceae & 3 & 5 & 2 & 2 & 8 & 20 \\
\hline Cola sp. & Sterculiaceae & 5 & 7 & 5 & 13 & 10 & 40 \\
\hline Combretum sp. & Combretaceae & 20 & 18 & 23 & 21 & 12 & 94 \\
\hline Commiphora africana & Burseraceae & 2 & 10 & 8 & 3 & 3 & 26 \\
\hline Corchorus olitorius & Malvaceae & 6 & - & - & - & - & 6 \\
\hline Costus afer & Costaceae & - & - & 3 & 2 & 5 & 10 \\
\hline Croton sp. & Euphorbiaceae & 9 & 3 & - & - & - & 12 \\
\hline Crossopteryx febrifuga & Rubiaceae & - & 3 & 7 & - & - & 10 \\
\hline Cucumis sp. & Cucurbitaceae & 2 & 4 & - & - & - & 6 \\
\hline Cyperaceae & Cyperaceae & 17 & 10 & 51 & 42 & 25 & 145 \\
\hline Daniellia oliveri & Caesalpinoideae & 9 & 1 & 8 & - & - & 18 \\
\hline Delonix regia & Caesalpinoideae & - & 10 & 6 & - & 4 & 20 \\
\hline Deterium microcarpum & Caesalpinoideae & - & - & - & 3 & 4 & 7 \\
\hline Diallium guineense & Caesalpinoideae & 3 & 3 & - & - & 21 & 27 \\
\hline Dichapetalum sp. & Dichapetaceae & - & - & 2 & 3 & - & 5 \\
\hline Dioscorea sp. & Dioscoreaceae & 2 & 3 & 1 & - & - & 6 \\
\hline Diospyros mellispiliformis & Ebanaceae & 9 & 1 & 2 & 10 & 35 & 57 \\
\hline Diospyros sp. & Ebanaceae & - & 2 & 4 & 2 & - & 8 \\
\hline Dissotis sp. & Melastomataceae & - & - & - & 11 & 26 & 37 \\
\hline Elaeis guineensis & Arecaceae & 395 & 302 & 320 & 350 & 173 & 1540 \\
\hline Empetrum nigrum & Caesalpinoideae & 3 & 2 & - & 2 & - & 7 \\
\hline Eucalyptus globus & Myrtaceae & - & - & - & - & 3 & 3 \\
\hline Euginea sp. & Myrtaceae & 10 & 27 & 32 & 13 & 10 & 92 \\
\hline Euphorbia hirta & Euphorbiaceae & 12 & 7 & 10 & 27 & - & 56 \\
\hline Euphorbia sp. & Euphorbiaceae & 3 & 3 & - & 1 & - & 7 \\
\hline Ficus exasperata & Moraceae & 3 & 1 & 2 & - & - & 6 \\
\hline Fillipendula ulmaria & Rosaceae & 30 & 22 & 59 & 12 & 4 & 127 \\
\hline Gaertnera paniculata & Rubiaceae & - & - & 4 & - & - & 4 \\
\hline Gardenia sp. & Rubiaceae & 3 & - & 2 & - & - & 5 \\
\hline Garcinia kola & Clusiaceae & - & 3 & - & 7 & 10 & 20 \\
\hline Gentianella amarella & Gentianaceae & - & - & - & 2 & 2 & 4 \\
\hline Gloriosa superba & Colchicaceae & 5 & 2 & 4 & 2 & - & 13 \\
\hline Gutenbergia sp. & Asteraceae & 26 & 28 & 54 & 118 & 40 & 266 \\
\hline
\end{tabular}




\begin{tabular}{|c|c|c|c|c|c|c|c|}
\hline Harungana madagascariensis & Hypericaceae & 5 & 9 & 20 & 16 & 19 & 69 \\
\hline Heliotropium sp. & Boraginaceae & - & - & 3 & - & - & 3 \\
\hline Hymenocardia acida & Phyllanthaceae & 20 & 31 & 81 & 8 & 7 & 147 \\
\hline Hyptis suaveolens & Lamiaceae & 27 & 9 & 10 & 4 & 15 & 65 \\
\hline Indigofera sp. & Papilionoideae & 6 & 3 & 2 & 5 & - & 16 \\
\hline Irvingia gabonensis & Irvingiaceae & 25 & 29 & 31 & 2 & 3 & 90 \\
\hline Jatropha curcas & Euphorbiaceae & - & 8 & - & - & 2 & 10 \\
\hline Justicia sp. & Acanthaceae & 4 & 1 & 3 & 4 & 1 & 13 \\
\hline Kedrostis africana & Cucurbitaceae & 2 & 6 & - & - & - & 8 \\
\hline Khaya senegalensis & Meliaceae & 23 & 45 & 9 & 1 & 8 & 86 \\
\hline Knautia sp. & Caprifoliaceae & - & - & - & - & 5 & 5 \\
\hline Lannea acida & Anacardiaceae & 75 & 113 & 71 & 68 & 33 & 360 \\
\hline Lasiodiscus sp. & Rhamnaceae & 9 & 7 & - & - & - & 16 \\
\hline Leea guineensis & Leeaceae & - & - & - & 2 & - & 2 \\
\hline Lepisanthes senegalensis & Diptilomoideae & 6 & 3 & - & - & - & 9 \\
\hline Leucas sp. & Lamiaceae & - & - & - & 2 & 9 & 11 \\
\hline Linum catharticum & Linaceae & 7 & 5 & 3 & 10 & 9 & 34 \\
\hline Linum sp. & Linaceae & 1 & 1 & 9 & 2 & 5 & 18 \\
\hline Lophira alata & Ochnaceae & 15 & 7 & 9 & 2 & - & 33 \\
\hline Luffa cylindica & Cucurbitaceae & - & 2 & 3 & - & - & 5 \\
\hline Mangifera indica & Anacardiaceae & - & 9 & 3 & - & - & 12 \\
\hline Manihot esculenta & Euphorbiaceae & 1 & 2 & 4 & 1 & - & 8 \\
\hline Merremia sp. & Convolvulaceae & 3 & - & - & 4 & - & 7 \\
\hline Microdesmis sp. & Pandaceae & 16 & 7 & 10 & - & - & 33 \\
\hline Milicia excelsa & Moraceae & 115 & 10 & 17 & 5 & 21 & 168 \\
\hline Mimosa pigra & Mimosoideae & 6 & 47 & 39 & 19 & - & 111 \\
\hline Morelia senegalensis & Rubiaceae & 143 & 6 & 12 & 5 & 21 & 187 \\
\hline Morus sp. & Moraceae & - & 4 & - & - & - & 4 \\
\hline Motandra sp. & Apocynaceae & 3 & 5 & 7 & - & - & 15 \\
\hline Nauclea latifolia & Rubiaceae & 1 & 27 & 54 & 16 & 10 & 108 \\
\hline Newbouldia laevis & Bignoniaceae & 41 & - & 13 & 8 & 11 & 73 \\
\hline Ocimum sp. & Lamiaceae & - & - & - & 3 & 5 & 8 \\
\hline Olax sp. & Olacaceae & 2 & - & - & - & - & 2 \\
\hline Parinari curetellifolia & Chrysobalanaceae & 31 & 5 & 11 & 9 & - & 56 \\
\hline Parkia biglobosa & Mimosoideae & 18 & 152 & 11 & - & - & 181 \\
\hline Paullinia pinnata & Sapindaceae & - & 7 & 25 & 16 & - & 48 \\
\hline Pentaclethra macrophylla & Mimosoideae & 37 & 25 & 11 & 8 & 2 & 83 \\
\hline Periploca sp. & Apocynaceae & 8 & 10 & 6 & 3 & 4 & 31 \\
\hline Phyllanthus sp. & Phyllanthaceae & 25 & 15 & 11 & 13 & 10 & 74 \\
\hline
\end{tabular}




\begin{tabular}{|c|c|c|c|c|c|c|c|}
\hline Physalis angulata & Solanaceae & - & 8 & - & 26 & 2 & 36 \\
\hline Piliostigma thonningii & Caesalpinoideae & 6 & 8 & - & - & 2 & 16 \\
\hline Pinus caribaea & Pinaceae & 1 & 4 & 4 & 1 & 2 & 12 \\
\hline Poaceae & Poaceae & 851 & 810 & 989 & 899 & 903 & 4,452 \\
\hline Prosopis africana & Mimosoideae & 59 & 22 & 7 & 19 & 8 & 115 \\
\hline Psidium guajava & Myrtaceae & - & - & - & 3 & - & 3 \\
\hline Pterocarpus osum & Papiloionoideae & 1 & 3 & - & 2 & - & 6 \\
\hline Pycnanthus angolensis & Myristicaceae & 5 & 2 & 1 & 2 & 3 & 13 \\
\hline Raphia sp. & Arecaceae & - & - & - & - & 2 & 2 \\
\hline Rauvolfia vomitoria & Apocynaceae & 2 & - & - & - & - & 2 \\
\hline Rhynchosia sp. & Papilionoideae & 5 & 4 & 3 & - & - & 12 \\
\hline Ricinus communis & Euphorbiaceae & - & 3 & - & 3 & - & 6 \\
\hline Securinega virosa & Phyllanthaceae & 5 & 2 & 3 & 5 & 5 & 20 \\
\hline Senna occidentale & Caesalpinoideae & 12 & 35 & 28 & 16 & 39 & 130 \\
\hline Sesamum indicum & Pedaliaceae & 2 & 4 & 1 & 2 & 8 & 17 \\
\hline Sida acuta & Malvaceae & 2 & 9 & 3 & 19 & 1 & 34 \\
\hline Solanum melongena & Solanaceae & 8 & 2 & 2 & 7 & 4 & 23 \\
\hline Solanum torvum & Solanaceae & 2 & 2 & 2 & - & - & 6 \\
\hline Spondias mombin & Anacardiaceae & 1 & 2 & - & 2 & - & 5 \\
\hline Sterculia sp. & Sterculiaceae & 5 & 2 & 3 & 5 & 5 & 20 \\
\hline Symphonia globulifera & Clusiaceae & 8 & 12 & 5 & - & - & 25 \\
\hline Syzygium guineense & Myrtaceae & 43 & 22 & 59 & 12 & 4 & 140 \\
\hline Talinum triangulare & Portulacaceae & - & 2 & 3 & - & - & 5 \\
\hline Tetracera sp. & Delleniaceae & 1 & 2 & - & - & - & 3 \\
\hline Tessmannia sp. & Caesalpinoideae & 3 & 7 & - & - & - & 10 \\
\hline Treculia africana & Moraceae & 5 & - & - & - & - & 5 \\
\hline Trichilia prieureana & Meliaceae & 3 & 7 & - & - & - & 10 \\
\hline Tridax procumbens & Asteraceae & 5 & 29 & 19 & 11 & 42 & 106 \\
\hline Triplochiton scleroxylon & Sterculiaceae & 20 & 40 & 46 & 8 & 7 & 121 \\
\hline Uapaca sp. & Phyllanthaceae & - & 4 & - & 6 & 2 & 12 \\
\hline Vigna unguiculata & Papilionoideae & 3 & - & - & - & - & 3 \\
\hline Vitellaria paradoxa & Sapotaceae & 5 & 10 & 5 & 2 & 5 & 27 \\
\hline Vitex grandifolia & Lamiaceae & - & - & 2 & 26 & 14 & 42 \\
\hline Zanthoxylum zanthoxyloides & Rutaceae & 3 & - & 5 & - & - & 8 \\
\hline Indeterminata & Indeterminata & 1 & 2 & 4 & 1 & 2 & 10 \\
\hline Sub total & & 2,664 & 2,338 & 2,587 & 2,130 & 1,923 & 11,642 \\
\hline \multicolumn{8}{|l|}{ Fern Spores } \\
\hline Anopteris hexagona & Pteridaceae & - & - & 3 & 1 & - & 4 \\
\hline Callistopteris apiifolia & Hymenophyllaceae & - & 2 & 3 & - & - & 5 \\
\hline
\end{tabular}




\begin{tabular}{llllllll}
\hline Cystopteris fragilis & Aspidiaceae & - & - & - & - & 2 & 2 \\
Goniopteris vivipara & Aspidiaceae & - & 9 & - & - & - & 9 \\
Monolete & & 43 & 36 & 39 & 36 & 23 & 177 \\
Nephrolepis exaltata & Davalliaceae & - & 11 & 6 & - & - & 17 \\
Pteris dentata & Pteridaceae & - & 2 & 2 & - & - & 4 \\
Trilete & & 4 & 2 & 5 & 7 & - & 18 \\
Sub total & 47 & 62 & 58 & 44 & 25 & 236 \\
\hline Other Palynomorphs & & & & & & \\
Algal cysts & 5 & 3 & 7 & 3 & 9 & 27 \\
Athropods/ parts & 140 & 468 & 51 & 168 & 274 & 1,101 \\
Bryophyte & 5 & 0 & 0 & 0 & 1 & 6 \\
Charred plant tissue & 457 & 124 & 396 & 133 & 184 & 1,294 \\
Diatom frustules & 149 & 276 & 125 & 186 & 199 & 935 \\
Dinoflagellate cysts & 9 & 13 & 7 & 5 & 0 & 34 \\
Fungal hyphae & 0 & 0 & 3 & 0 & 0 & 3 \\
Plant tissues & & 0 & 0 & 1 & 0 & 7 & 8 \\
Sub-total & 765 & 884 & 590 & 495 & 674 & 3408 \\
\hline Grand total & 3,476 & 3,284 & 3,235 & 2,669 & 2,622 & 15,286 \\
\hline
\end{tabular}

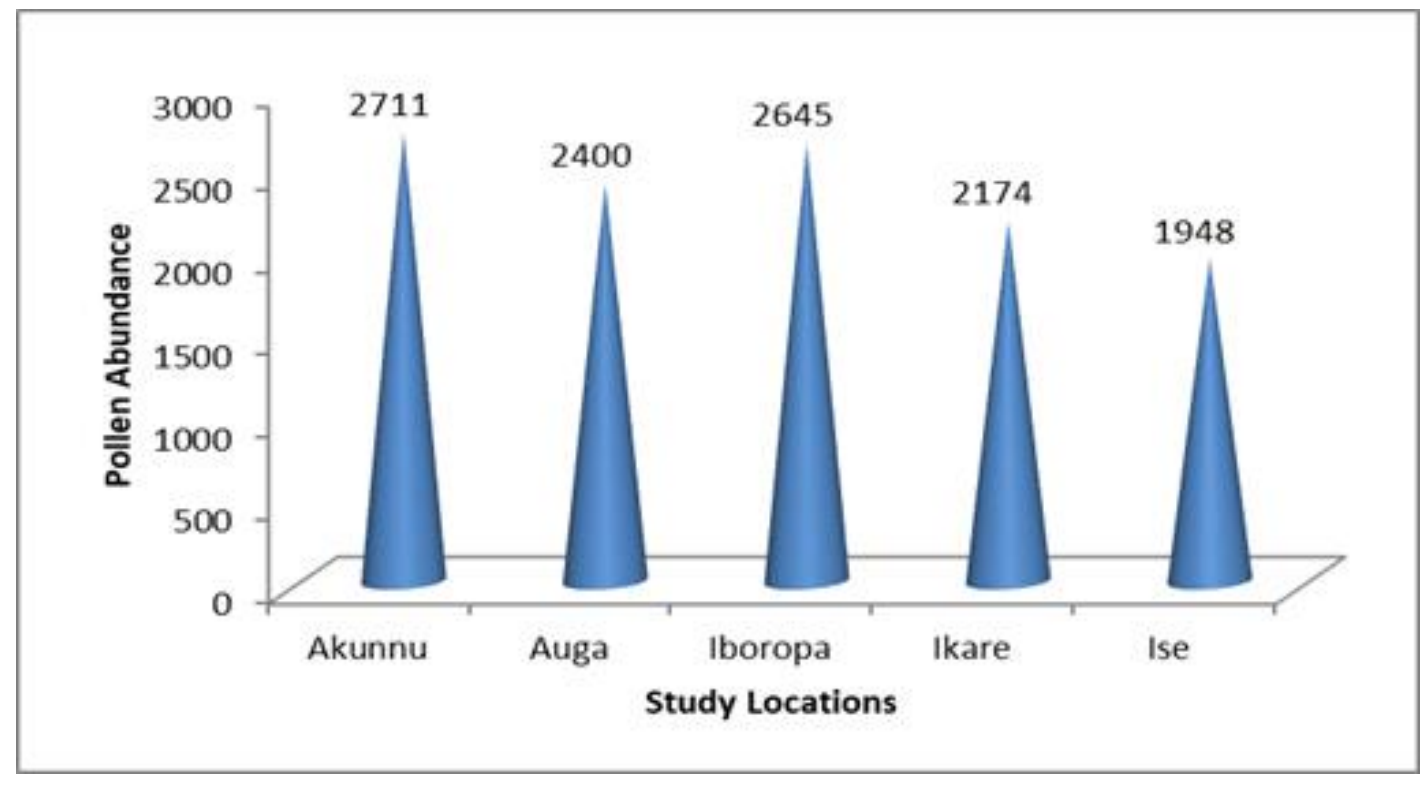

Figure 1 Distribution of palynomorphs abundance across the study location 
Ige and Essien / GSC Biological and Pharmaceutical Sciences 2019, 08(01), 064-077

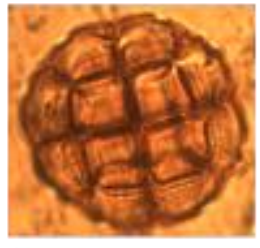

Albizio zygia

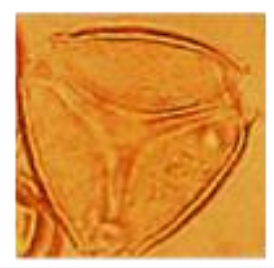

Aubrevillea kerstingii

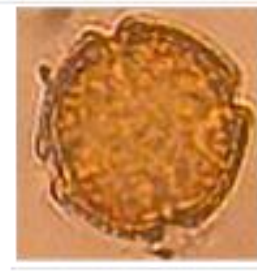

Dissotis sp.

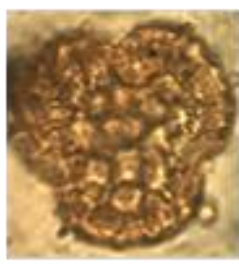

Delonix reqia

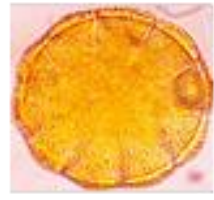

Ocimum sp.

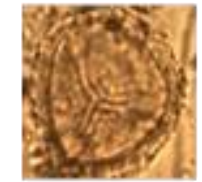

Trilete
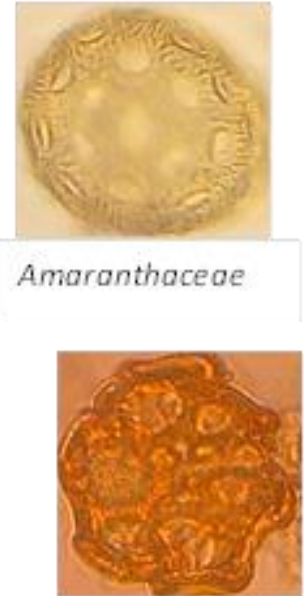

\section{Brachystegia eurycoma}

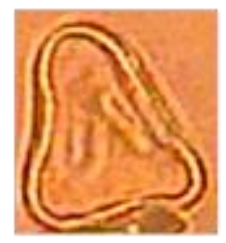

Citrus sp.

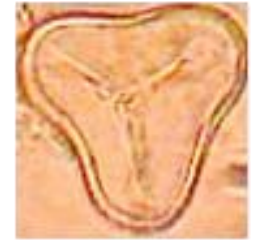

Elaeis guineensis

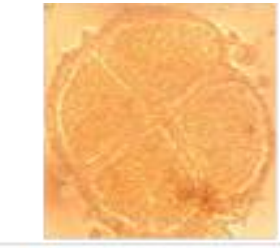

Annona senegalensis

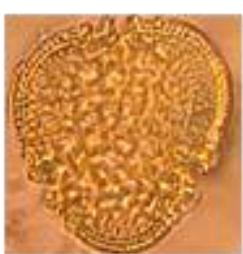

Ceiba pentandra

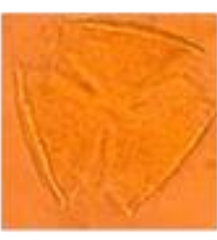

Cyperaceae

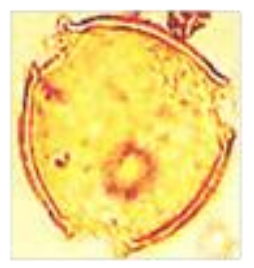

Knautia sp.

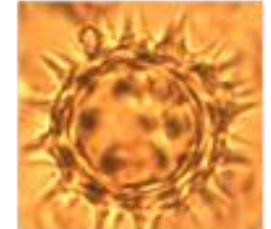

Aspillia africana

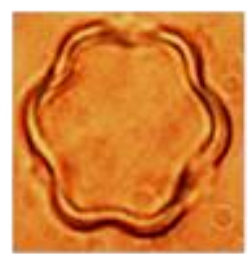

Combretumsp.

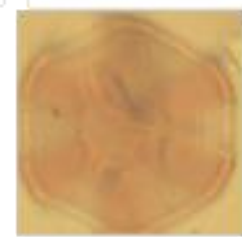

Daniella oliveri

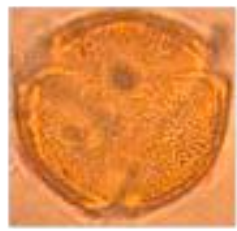

Linum sp.

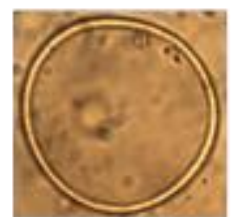

Poaceae

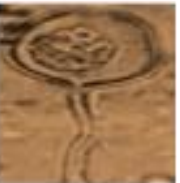

Algal cysts

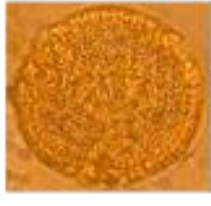

Pycnanthus

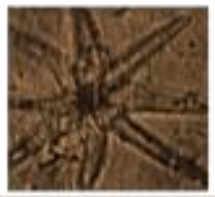

Bryophyte
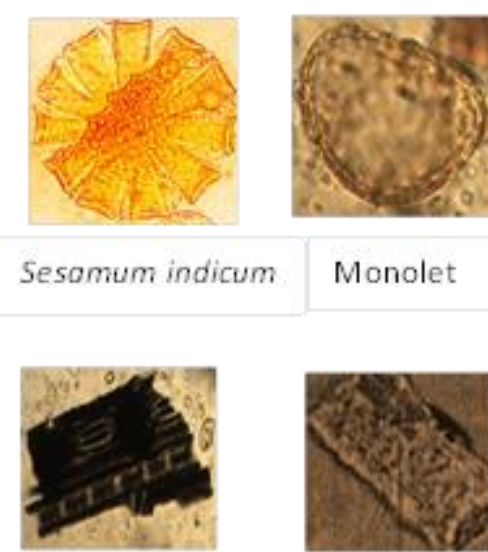

Charred plant

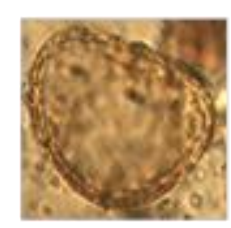

Monolet

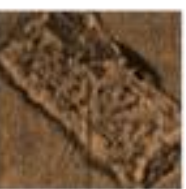

Diatom frustules

Figure 2 Photomicrograph of selected Palynomorphs 
Table 3 Mean pollen grains recorded at the study locations

\begin{tabular}{lll}
\hline S/N & Study Location & Mean pollen grains \pm S.E \\
\hline 1 & Ise & $0.86 \pm 0.17^{\mathrm{a}}$ \\
2 & Ikare & $0.94 \pm 0.18^{\mathrm{b}}$ \\
3 & Auga & $1.05 \pm 0.17^{\mathrm{c}}$ \\
4 & Iboropa & $1.14 \pm 0.19^{\mathrm{abc}}$ \\
5 & Akunnu & $1.19 \pm 0.18^{\mathrm{abc}}$ \\
& p Value & $0.000^{*}$ \\
\hline \multicolumn{2}{l}{ Means not followed by the same letter are significantly different at $\mathrm{P}<0.05$ (DMRT). } \\
\end{tabular}

The predominant pollen types include those of Poaceae, Elaeis guineensis, Alchornea cordifolia, Aspilia africana, Casuarina equisetifolia, Tridax procumbens, Lannea acida, and Gutenbergia species.

\subsection{Elaeis guineensis}

Pollen grains with absolute count of 1,540 were encountered in the study. Elaeis guineensis which is a major pollen contributor in this study is found in all forms of sub-types vegetation in Akoko environment, Ondo State ranging from urban flora to grasslands, farmlands, woodlands and mature nature forests. Akoko people are known for the cultivation and production of oil palm and palm produce. Because of their enormous economic benefits, they are protected and established in farms and plantations. They flower virtually all year round, hence its characteristic increase and decrease in pollen content; turning out large quantities of pollen grains particularly at the major flowering periods. Its presence in the pollen spectra of the study area depicts extension of wooded grassland and traditional forest.

The aerodynamic properties of the pollen grains promote their dispersal and distribution in the turbulent air mass. This accounts for part of the reason why the pollen grains of Elaeis guineensis were trapped in the atmosphere throughout the twelve (12) months of study and at each each sampling site at least in reasonable proportion. Findings corroborated favourably with the report of [24].

\subsection{Poaceae}

Pollen encountered in this study totaled 4,452. The grass family was reported to be the major contributors to airborne pollen assemblage in most aeropalynological studies [5, 8, 9, 13, 25]. Mabberley [26] in Latorre and Belmonte [27] reported that the Poaceae family has a cosmopolitan distribution representing $20 \%$ of the world's vegetation cover. With the light microscope, it is difficult to separate the Poaceae pollen on the species level. For this reason, all Poaceae pollen grains are grouped together.

However, the increase of grass pollen could also be attributed to the fact that most members of these family flowers and shade their floral parts and subsequent shriveling of these flowers which eventually makes the entire plants to dry up as the environment approaches extreme dryness and destruction through annual bush burning thereby liberating their pollen into the atmosphere.

Similar results of high Poaceae pollen counts have been obtained by previous researchers [5, 6, 28, 29, 30, 31, and 32].

\subsection{Alchornea cordifolia}

Contributed about 392 pollen grains in the airborne pollen spectrum of Akoko North-East Local Government Area of Ondo State, Nigeria. Alchornea cordifolia is associated with human activities in the studied environment and also as early colonizers of deforested environment. The human impact on the tropical rainforest probably fostered the growth of Alchornea cordifolia and the presence of its pollen reflects the impact of anthropogenic activities on the study environment. The high record of its pollen grains is attributed partly to the anemophilous mode of pollination and the buoyant capacity of the pollen grains [24]. 


\subsection{Casuarina equisetifolia}

Casuarina equisetifolia is an exotic plant and produces abundant pollen grains (269) in this study. It is grown in most places as ornamental tree and the pollen has been widely recorded in most aeropalynological studies in Nigeria in addition to those of Poaceae, Elaeis guineensis, Alchornea cordifolia and members of the family Asteraceae among others [30, 33, 34, 35, 36, 37]. It could also be pointed out that the relative abundance of the pollen of most of these plants in all the location is not only as a result of their copious pollen producing capacity and anemophilous mode of pollination, but their general distribution at all the sampling locations.

Though most of the pollen types encountered from the study reflected to a greater extent the flora of Akoko environment, the presence of pollen of Empetrum nigrum, Linum sp. and Vitellaria paradoxa is a valid evidence of long distance transport by the North East trade wind from Europe and the West Mediterranean region southwards and the action of meteorological factors in the transportation and dispersal of these pollen grains away from their source locality to another area of the same and/ or different ecological characteristics since the plant has not been sited anywhere in the study (locations) environment for example.

\subsection{Empetrum nigrum}

Empetrum nigrum contributed about 7 pollen grains in this study. Evolutionary biologists have explained the stricking geographic distribution of Empetrum nigrum as a result of long distance migratory bird dispersing seeds from one pole to the other [38].

\subsection{Linum}

Linum species contributed about 52 pollen grains in this study and are native to temperate and subtropical regions of the world while Vitellaria paradoxa formally known as Butryospermum paradoxum grows abundantly in the northern Guinea Savanna and contributed about 27 pollen grains in this study. The presence of their pollen in the airborne palynomorphs of the studied environment can be attributed to the impact of long distance transport.

\subsection{Pteridophytes}

Spore of pteridophytes constitutes a very low proportion of the total palynomorphs encountered in the study (Tables 1 and 2). Due to identification problems, only six fern spore types were identified to generic level while majority of the spores were classified under monolete and trilete. A total of 236 fern spores were recorded. The dominant ones are Goniopteris vivipara and Callistopteris apiifolia. The monolete spores (177) were more abundant than the trilete spores (18). The results presented in this study showed that a considerable quantity of the fern spores were recorded at the locations Auga (62), Iboropa (58), Akunnu (47), Ikare (44) and Ise (25) respectively during the early rainy season, but declined with the increase in rainfall from July which may be compared to the dry season where only a few ferns sporulate due to unfavourable conditions [39]. Nevertheless, the unusual increase in the abundance of fern spores in the months of October, September and November respectively could be attributed to the action of meteorological factors for example wind; which waft up the dried and already deposited dry airspora into the air current (wave) causing an unusual increase in the atmosphere.

\subsection{Charred plant particles}

These are charred particles of grasses, weeds, herbs and trees released during domestic or wild fire incidence that are wafted into the atmosphere. They mostly constitute of charred cuticles, epidermal layers, cells and other plant tissues. A total of 8 plant tissues and 1,294 charred plant particles were documented in this study (Tables 1 and 2). A reasonable quantity of them was trapped at all the locations and was recorded from the air throughout the study duration especially during the dry period of the year. They serve as indicator of bush fire and can be used in monitoring the intensity and frequency of bush fires. Findings agree favourably with the report of Agwu [6] and Morley and Richards [40] who demonstrated the application of 'Charred Graminae Cuticle' as a key identification of late Cenozoic climate changes in the Niger Delta of Nigeria.

\subsection{Diatom frustules}

The Diatom frustules were among the consistent particles recorded in this study and they represent an aspect of algal microflora in Akoko environment. A total of 935 Diatom frustules were encountered in this study (Table 1). They occurred in reasonable quantities throughout the period of study and were recorded at all the locations and may be associated with dry ponds, drying of seasonally flooded places and loose dry soils that provide habitat to these algae. During prolonged dryness in Akoko North-East environment, ponds and other water logged areas dry up and expose the fresh water algae (diatoms) to dust storms/ strong harmattan winds. The whirl winds waft up the dried mud into 
the air current causing an unusual increase in the atmosphere. Their increase in the airspora of Akoko North-East environment is an indication of increasing dryness and the arrival of long distance transported materials from Northeast (NE) trade wind otherwise known as Harmattan. Findings corroborated favourably with the report of Nwankwo [41]. In environmental analysis, diatoms are useful palaeoecological indicators and have distinct ecological tolerance; and they provide a substantial amount of autecological information [42].

Among the other airborne palynomorphs encountered in this study are dinoflagellate cysts. A total of 34 dinoflagellate cysts were documented (Table 1). Few particles of fungal hyphae were recorded representing the enormous fragments circulating in the atmosphere. Fungal hyphae are among the common palynomorphs trapped in most aeropalynological studies. A total of 3 fungal hyphae were encountered in this study and they serve as inocula for fungal infection or as saprophytes (Table 1).

Also, some bryophyte particles were distinguished and recorded from the trapped particles. Other remains distinct from the charred ones and was also recorded as part of the bioparticles circulating in the atmosphere. Bryophyte remains, algal cysts and uncharred plant tissues (particles) recorded in this study may serve interpretational purposes in environmental reconstruction. Similar findings have been reported by Agwu and Osibe [43] and Njukuocha and Osayi [44].

\section{Conclusion}

Monitoring the pollen content of the air has become a standard practice. The results showed that the predominant pollen types belong to the family Poaceae, Elaeis guineensis, Alchornea cordifolia, Aspilia africana, Casuarina equisetifolia, and Tridax procumbens. The presence of Empetrum nigrum, Linum sp. and Vitellaria paradoxa pollen indicates evidence of long distance transport. There were significant differences in the mean pollen counts depending on the location and time of collection. Rainfall and relative humidity had more negative effect on pollen concentration. The effects of temperature and light intensity varied considerably with time and location because their effects are influenced by other meteorological factors. Airborne pollen grains were most abundant between September and January. Generally. The pollen types identified in this study reflect to a large extent the phytoecological vegetation of the study environment. The various ecological indicator species identified confirmed their origin as coming from forest-savanna ecozone that is anthropogenically disturbed.

\section{Compliance with ethical standards}

\section{Acknowledgments}

The authors are grateful to the Almighty God for life, protection and inspirations; and to themselves for the financial commitment towards the success of this research.

\section{Disclosure of conflict of interest}

The authors declare that there is no conflict of interest.

\section{References}

[1] Roberts N. (1998). The holocene: an environmental history. Basil-Blackwell, New York, 350 - 361.

[2] Erdtman G. (1969). Handbook of Palynology. Muntisguard, Copenhagen, 280 - 285.

[3] Moore PD and Webb JA. (1978). An illustrated guide to pollen analysis, First edition. Hodder and Stoughton, Kent-London, 133- 133.

[4] Hopping CA. (1967). Palynology and the oil industry. Review of Palaeobotany and Palynology, 2, 23-48.

[5] Essien BC. (2014). A comparative analytical study of airborne pollen grains, spores and other palynomorphs within Grimard Catholic hospital, Anyigba, Kogi State, Nigeria. Scholarly Journal of Scientific Research and Essay, 3(5), 56 - 60.

[6] Agwu COC. (2001). A Study of Niger Delta environment through air-borne palynomorphs, Port-Harcourt, Nigeria. Palaeoecology of Africa, 27, 191- 205. 
[7] Lezine AM. (1988). New pollen data from the Sahel, Senegal. Review of Palaeobotany and Palynology, 55, 141154.

[8] Njokuocha RC. (2006). Airborne pollen grains in Nsukka, Nigeria. Grana, 45, 73-80.

[9] Njokuocha RC and Agwu COC. (2007). Airborne fungal spores in Nsukka municipality. Nigerian Journal of Botany, 20(2), $349-359$.

[10] Amule OH. (2008). Palynological studies of quaternary sediments from Lagos lagoon and its environments. Ph.D. Dissertation, University of Lagos, Nigeria. 4 -276.

[11] Ige OE. (2009). A late tertiary pollen record from Niger Delta, Nigeria. International Journal of Botany, 5(3), 203 -215 .

[12] Ige OE. (2011). Vegetation and climate history of the late tertiary Niger Delta, Nigeria, based on pollen record. Research Journal of Botany, 6, 21-30.

[13] Essien BC and Agwu COC. (2013). Aeropalynological study of Anyigba, Kogi State, Nigeria. Standard Scientific Research Essays, 1(13), 347-351.

[14] Patton MQ. (1990). Quantitative evaluation and research methods, Second edition. Sage Publications, Newbury Park, CA, 1- 523.

[15] Tauber H. (1974). A static non-overload pollen collector. New Phytology, 73, 359 -369.

[16] Tauber H. (1977). Investigations of aerial pollen transport in a forested area. Dansk Botanisk Arkiv, 32(1), 1 121.

[17] Pardoe HS, Giesecke T, van der Knaap WO, Svitavaska-Svobodova H, Kvavadze EV, Panajiotiotidis S, Gerasimidis A, Pidek IA, Zimney M, Swieta-Musznicka J, Latalowa M, Noryskiewiez AM, Bozilova E, Tonkov S, FilipovaMarinova MV, van Leeuwen JFN and Kalnipa L. (2010). Comparing pollen spectra from modified Tauber traps and moss samples: example from a selection of woodlands across Europe. Vegetational History and Archaeobotany, 19, $271-283$.

[18] Giesecke T, Fontana SL, van der Knaap WO, Pardoe HS and Pidek IA. (2010). From early pollen trapping to experiments to the pollen monitoring programme. Vegetational History and Archaeobotany, 19, $247-258$.

[19] Agwu COC and Akanbi TO. (1985). A palynological study of honey from four vegetation zones of Nigeria. Pollen et Spores, 27, 335-348.

[20] Bonnefille R and Riollet G. (1980). Pollen des savanna d'Afrique orientale. Anatole Press, Paris, France, 1 - 140.

[21] Kremp GOW and Kawasaki T. (1972). The spores of Pteridophytes. Illustration of the spores of the ferns and fern allies. Hirokawa Publishing Company Incorporated, Tokyo, Japan, 1 - 398.

[22] Sowunmi MA. (1995). Pollen of Nigerian plants II. Grana, 34, 120 -141.

[23] Shubharani R, Roopa P and Sivaram V. (2013). Pollen morphology of selected bee forage plants. Global Journal of Bio-Science and Biotechnology, 2(1), 82 - 90.

[24] Njokuocha RC. (2012). Factors affecting the distribution and variation of airborne palynomorphs in Enugu State, Nigeria. Ph.D. Dissertation, University of Nigeria, Nsukka, 16 -121.

[25] Adeniyi TA, Adeonipekun PA and Olowokedujo JD. (2018). Comparative study of aeropollen and pollinosis cases. Environmental Monitoring and Assessment, 190, 562 -572.

[26] Mabberley DJ. (1987). The plant book. Cambridge University Press, Cambridge.

[27] Latorre F and Belmonte J. (2004). Temporal and spatial distribution of atmospheric poaceae pollen in Catalonia (NE Spain) in 1996-2001. Grana, 43, $156-163$.

[28] Al-Eisawi D and Danjani B. (1988). Airborne pollen of Jordan. Grana, 27, 219 -227.

[29] Mishra RP, Singh B and Oommachan M. (2002). Airborne pollen flora of Jabulur- the Central India. Aerobiologia, $18,73-81$.

[30] Njokuocha RC and Ezenwajiaku, TE. (2010). Airborne pollen flora in Aguata Local Government Area, Anambra State, Nigeria. Nigerian Journal of Botany, 23(2), 211- 224. 
[31] Adeonipekun PA, Agbalaya AE and Adeniyi TA. (2016). Aeropalynology of Ayetoro-Itele, Ota, South-West Nigeria: a preliminary study. In: Alabi R and Adeonipekun PA (Eds), Human Palaeoecology in Africa: Essays in Honour of M. Adebisi Sowunmi. University of Ibadan Press, Ibadan, Nigeria, 130 -153.

[32] Adeniyi TA, Adeonipekun PA, Olowokedujo JD and Akande IS. (2018). Allergenicity of dominant aeropollen in Nigeria, part II. Current Allergy and Clinical Immunology, 31(3), 178 -183.

[33] Agwu COC. (1997). Modern pollen rain in Nsukka: An indicator of the vegetation of Nsukka Plateau. Wurzburger Geographische Arbeiten, 92, 97 -115.

[34] Agwu COC, Njokuocha RC and Mezue 0. (2004). The study of airborne pollen and spores circulating at "head level" in Nsukka environment. Bio-Research, 2(2), 7 -14.

[35] Njokuocha RC and Ukeje HO. (2006). The study of airborne pollen precipitation in the University of Nigeria (Nsukka) Botanic Garden. Bio-Research, 4(2), 88 - 93.

[36] Adeonipekun PA. (2012). Comparative aeropalynology of same months of two consecutive years in AyetoroItele, Ota, South-West Nigeria. JENE, 4(12), 303 -313.

[37] Adeniyi TA, Adeonipekun PA, Olowokedujo JD and Akande IS. (2014). Airborne pollen records of Shomolu Local Government Area in Lagos State. Notulae Scientia Biologicae, 6(4), 428-432.

[38] Magnus P, Virginia M and Christian B. (2011). A single mid-pleistocene long-distance dispersal in Empetrum nigrum. Missouri Botanical Garden, 108 (16), 6520 - 6525.

[39] Hutchinson J and Dalziel JM. (1954-72). Flora of West Tropical Africa, Volume 1, 2, and 3. Whitefriars, London, 1 - 1399.

[40] Morley RJ and Richards K. (1993). Grammineae cuticle: a key indicator of Late Cenozoic climate change in the Niger Delta. Review of Palaeobotany and Palynology, 77, 119-127.

[41] Nwankwo DI. (1990). Contribution to the diatom flora of Nigeria 1 - diatoms of Lagos lagoon and the adjacent sea. Nigerian Journal of Botany, 3, 53 -69.

[42] Ige OE and Essien BC. (2019). Monitoring environmental change in Akoko North-East Local Government Area of Ondo State, Nigeria through pollen analysis. A paper presented at the 10th Palynological Association of Nigeria (PAN) Conference. Adekunle Ajasin University, Akungba-Akoko, Ondo State, Nigeria. 5th to 9th May, 2019.

[43] Agwu COC and Osibe EE. (1992). Airborne palynomorphs of Nsukka during the months of February-April, 1990. Nigerian Journal of Botany, 5, 177-185.

[44] Njokuocha RC and Osayi EE. (2005). Airborne pollen survey in relations to allergy and plant pathogens in Nsukka, Nigeria. Nigerian Journal of Biological Research and Biotechnology, 3(1), 77 - 84.

\section{How to cite this article}

Ige OE and Essien BC. (2019). The applications of pollen analysis in environmental monitoring in Akoko North-East Local Government Area of Ondo State, Nigeria. GSC Biological and Pharmaceutical Sciences, 8(1), 64-77. 